FTPI-MINN-05/39

September 2005

\title{
Hidden Modulus in the Extended Veneziano-Yankielowicz Theory
}

\author{
Roberto Auzzi $^{a}$ and Francesco SAnnino ${ }^{b}$ \\ ${ }^{a}$ William I. Fine Theoretical Physics Institute, University of Minnesota, \\ Minneapolis, MN 55455, USA \\ ${ }^{b}$ The Niels Bohr Institute, Blegdamsvej 17, Copenhagen Ø, Denmark.
}

\begin{abstract}
The issue of domain walls in the recently extended VenezianoYankielowicz theory is investigated and we show that they have an interesting substructure. We also demonstrate the presence of a noncompact modulus. The associated family of degenerate solutions is physically due to the presence of a valley of vacua in the enlarged space of fields. This is a feature of the extended Veneziano-Yankielowicz theory. Unfortunately the above properties do not match the ones expected for the domain walls of $\mathcal{N}=1$ super Yang-Mills.
\end{abstract}




\section{Introduction}

Pure $\mathcal{N}=1 S U(N)$ super Yang-Mills theory is known to possess $N$ independent vacua. The issue of the domain walls between these vacua is very interesting. These objects are expected to saturate the central charge of $\mathcal{N}=1$ superalgebra [1] and the tension of the wall between the 1 -st and the $\mathrm{k}$-th vacua is [2]:

$$
T_{k} \propto \sin \frac{\pi k}{N}
$$

Moreover, there are arguments from string theory [3] and also from field theory [4, 5] which suggest that the walls have the following multiplicity:

$$
n_{k}=\frac{N !}{k !(N-k) !} \text {. }
$$

Unfortunately a dynamical description of these domain walls using the basic fields of super Yang-Mills or the composite ones is still lacking. In the framework of the Veneziano-Yankielowicz (VY) effective lagrangian it is not possible to find a wall solution interpolating the different vacua [10, 11, 12, 13, 14. We remind the reader that the VY theory concisely summarizes the symmetry of the underlying theory in terms of the following composite chiral superfield

$$
S=\frac{3}{32 \pi^{2} N} \operatorname{Tr} W^{2}
$$

where $W_{\alpha}$ is the supersymmetric field strength. When interpreting $S$ as an elementary field it describes a gluinoball and its associated fermionic partner.

The Kähler potential and the superpotential are respectively [6]

$$
K(S, \bar{S})=\frac{9 N^{2}}{\alpha}(S \bar{S})^{\frac{1}{3}}, \quad W_{V Y}[S]=\frac{2 N}{3} S \log _{(0)}\left(\frac{S}{e \Lambda^{3}}\right)^{N}
$$

where $\alpha$ is a dimensionless real parameter and we restricted ourselves to the first branch of the logarithm. The complex bosonic degree of freedom $\phi_{s}$

in the VY Lagrangian posses the same quantum numbers of the gaugino bilinear $\lambda \lambda$. The latter is expected to condense spontaneously breaking the $Z_{2 N}$ symmetry to $Z_{2}$ and leaving behind $N$ vacua.

On general grounds there are in principle two possibilities for the domain walls; the wall can pass in the real $S$ space through the value $\phi_{s}=0$ or it can 
pass away from this value in the complex $\phi_{s}$ plane. The real wall solution is somehow a very particular one: it can interpolate only between two vacua aligned with the origin. This implies that we can have a real wall passing through the origin only when $N$ is even and $k=N / 2$. Then there will be $N / 2$ pairs of vacua aligned with the origin. In the VY Lagrangian approach there are problems for both the possibilities: the real wall solution stops at the origin at $\phi_{s}=0$ and so cannot interpolate between the two vacua at $\phi_{s}= \pm \phi_{s 0}$; for the complex wall there are problems due to the logarithmic cuts in the superpotential.

It is natural at this point to study the domain wall problem in the recently proposed extension of the VY effective Lagrangian [7]. Real domain walls solutions were found in this approach in Ref. [9]; in this work we further investigate these domain wall solutions.

We will study the two color theory, however our results are generally applicable to an even number of colors for the domain wall solutions interpolating between two vacua aligned with the origin. We will demonstrate that the domain wall solutions possess a hidden noncompact modulus. The presence of such a modulus is due to the fact that there exists a valley of vacua at the origin in the enlarged space of fields with respect to the original VY theory which contains only one chiral superfield.

In the next section we briefly introduce the extended VY theory while in section three we show how a modulus emerges when investigating the real domain walls in this theory. In section four we briefly discuss the issue of complex walls. We finally conclude in section five and provide also some outlook.

\section{Extended VY Theory}

There are many reasons to consider extending the VY theory. When trying to break SYM to YM one would expect the glueball degrees of freedom to be present, for example. However in the original theory such states are missing. Many different approaches were considered in the literature to extend the VY theory and the reader can find an exhaustive list of references in [7]. In [7. a number of different consistency checks was shown to naturally lead to the following form of the extended VY superpotential:

$$
W(S, \chi)=\frac{2 N^{2}}{3} S\left[\log _{(0)} S-1-\log \left(-e \frac{\chi}{N} \log _{(0)} \chi^{N}\right)\right] .
$$


A Kähler potential is needed to investigate various dynamical properties. The simplest one for $S$ which is consistent with the quantum anomalies in the VY theory is $(S \bar{S})^{1 / 3}$. Due to the presence of the new field $\chi$ one can modify the Kähler potential in order to provide a kinetic term also for $\chi$ :

$$
K(S, \bar{S}, \chi, \bar{\chi})=\frac{9 N^{2}}{\alpha}(S \bar{S})^{1 / 3} h(\chi, \bar{\chi})
$$

where $h(\chi, \bar{\chi})$ is a real positive definite function. This is the generic form

of the Kähler potential in order not to upset the saturation of quantum anomalies. The associated Kähler metric is:

$$
g_{i \bar{j}}=\frac{\partial^{2} K}{\partial \phi^{i} \partial \bar{\phi}^{\bar{j}}} .
$$

The potential is:

$$
V=\frac{\partial W}{\partial \phi^{i}} g^{i \bar{j}} \frac{\partial \bar{W}}{\partial \bar{\phi}^{j}},
$$

where

$$
g^{i \bar{j}}=\left(g_{i \bar{j}}^{-1}\right)^{T} .
$$

In reference [8] it was argued that the glueball states are heavier than the gluinoball states. If this were not the case there would have been no reason to saturate the underlying anomalies of the theory using only the composite objects constructed out of the gluinoball fields. Since the extended VY theory well describes the vacuum properties of SYM and the spectrum of the lowest lying states of the theory one would hope that it can also overcome the problems related to the domain wall solutions and their multiplicity.

\section{Real BPS walls: A new moduli space}

Recently the domain walls in the extended VY for $N=2$ were investigated in Ref. 9, where real domain wall solutions were found to exist. We recall the general BPS domain wall equations for a generic supersymmetric WessZumino model with more than one field labeled by Latin letters:

$$
\frac{d \phi^{i}}{d z}=e^{-i \beta} g^{i \bar{j}} \partial_{\bar{j}} \bar{W}
$$


We have only two scalar fields $\phi^{1}=\phi_{S}$ and $\phi^{2}=\phi_{\chi}$. The system of differential equations reduces to (see Ref. [9])

$$
\frac{\partial}{\partial z}\left(\begin{array}{c}
\varphi \\
\varphi_{\chi}
\end{array}\right)=\frac{\alpha}{N^{2}} \frac{(\bar{\varphi} \varphi)^{\frac{2}{3}}}{h h_{\chi \bar{\chi}}-h_{\chi} \bar{h}_{\bar{\chi}}}\left[\begin{array}{cc}
h_{\chi \bar{\chi}} & -\frac{\bar{\varphi}^{-1} h_{\chi}}{3} \\
-\frac{\varphi^{-1} \bar{h}_{\bar{\chi}}}{3} & \frac{h}{9}(\bar{\varphi} \varphi)^{-1}
\end{array}\right]\left(\begin{array}{c}
\partial_{\bar{S}} \bar{W} \\
\partial_{\bar{\chi}} \bar{W}
\end{array}\right),
$$

with $h_{\chi}=\partial_{\chi} h, \bar{h}_{\bar{\chi}}=\partial_{\bar{\chi}} h$ and $h_{\chi \bar{\chi}}=\partial_{\chi} \partial_{\bar{\chi}} h$. We also have

$$
\partial_{S} W=\frac{2 N^{2}}{3} \log _{(0)}\left(\frac{S}{-e \frac{\chi}{N} \log _{(0)} \chi^{N}}\right), \quad \frac{\partial_{\chi} W}{S}=\frac{2 N}{3} \frac{\log _{(0)} \chi^{N}+N}{-\frac{\chi}{N} \log _{(0)} \chi^{N}} .
$$

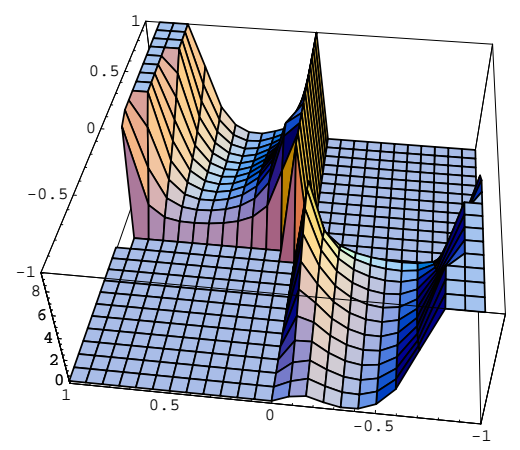

Figure 1: Potential $V\left(\phi_{s}, \phi_{\chi}\right)$ for $N=2$ and for the Kähler $h_{2}$ for real $\phi_{s}, \phi_{\chi}$. It goes to infinity at $\phi_{\chi}=0$ and at $\phi_{\chi}= \pm 1$; there is a valley of zero potential at $\phi_{s}=0$.

The potential $V$ for real $\phi_{s}, \phi_{\chi}$ can be written in the following way:

$$
V\left(\phi_{s}, \phi_{\chi}\right)=\left|\phi_{s}\right|^{4 / 3}\left(f\left(\phi_{\chi}\right) \log \left|\phi_{s}\right|^{2}+g\left(\phi_{\chi}\right)\right)
$$

where $f, g$ are two functions of $\phi_{\chi}$ depending on $h(\chi, \bar{\chi})$ in Eq. (44). The potential $V$ has a valley of zeros at $\phi_{s}=0$. This valley of vacua exists for every function $h$ in Eq. (4) and so it is a general property of the extended VY Lagrangian (see Fig. 1). Actually the point $\phi_{s}=\phi_{\chi}=0$ is not part of this valley of vacua. The potential assumes different values according to the path one uses when approaching the origin.

The existence and behavior of the walls depend not only on the superpotential of the effective theory, but also on the Kähler potential. In Ref. 9] the following Kähler potentials were considered and motivated:

$$
h_{1}(\chi, \bar{\chi})=\left(1+\gamma e^{2} \chi \bar{\chi}\right)\left(e^{2} \frac{\chi \bar{\chi}}{N^{2}} \log \chi^{N} \log \bar{\chi}^{N}\right)^{-\frac{1}{3}},
$$


and

$$
h_{2}(\chi, \bar{\chi})=\left(1+\gamma e^{2} \chi \bar{\chi}\right)\left(e^{2} \chi \bar{\chi}\right)^{-\frac{1}{3}} .
$$

Explicit solutions interpolating two opposite vacua $\left(\phi_{s}, \phi_{\chi}\right)=\left( \pm \phi_{S 0}, \pm \phi_{\chi 0}\right)$ were found for both the Kähler potentials. These solutions pass through the origin in the field space $\left(\phi_{s}, \phi_{\chi}\right)=(0,0)$. We are going to present a detailed analysis for the theory assuming the Kähler potential $h_{2}$; the case in which one uses $h_{1}$ is analogous. The reason is that the two Kählers have a similar behavior near the origin $\phi_{s}, \phi_{\chi}=0$.
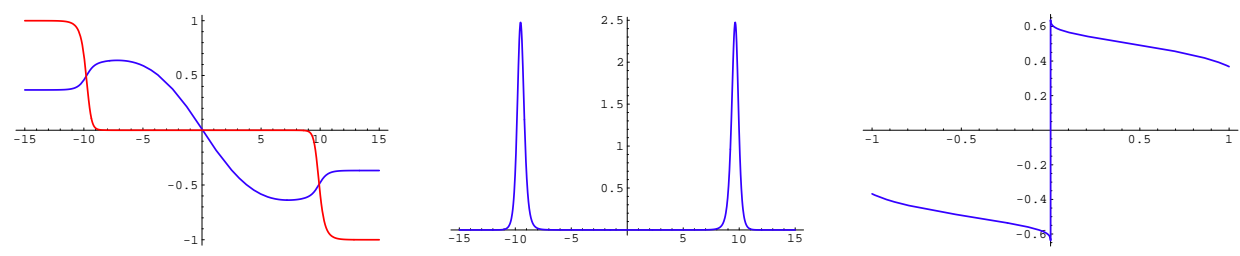

Figure 2: Wall for the Kähler potential $h_{2}$ and at large $l$. Left: Field profiles for $\phi_{s}(z)$ and $\phi_{\chi}(z)$. Center: Energy density. Right: Field profiles in the plane $\left(\phi_{s}, \phi_{\chi}\right)$. The fields pass through the valley at $\phi_{s}=0$.

We have tried to generalize the analysis to the following class of Kähler potentials:

$$
h(\chi, \bar{\chi})=\left(1+\gamma e^{2} \chi \bar{\chi}\right)\left(e^{2} \chi \bar{\chi}\right)^{p} .
$$

If we keep only the dominant terms near the origin we find the following equation:

$$
\frac{d \phi_{s}}{d \phi_{\chi}}=-3 p \frac{\phi_{s}}{\phi_{\chi}},
$$

which has a regular solution near the origin only if $-3 p$ is a positive integer:

$$
\phi_{s}=C \phi_{\chi}^{-3 p}
$$

We have explored different values of $p$. However it turns out that the only acceptable value, in order to have a well behaved domain wall solution, is $p=-1 / 3$.

A solution of the wall equation interpolating between the vacua at $\phi_{s}=$ $\pm \phi_{s 0}$ can pass through the valley of vacua at $\phi_{s}=0$; so it can be thought as composite of two walls, one interpolating between $\phi_{s}=\phi_{s 0}$ and $\phi_{s}=0$ and the other interpolating $\phi_{s}=0$ and $\phi_{s}=-\phi_{s 0}$. Hence, there is a hidden 
modulus which describes the distance $l$ between the two component walls. The parameter $l$ can be defined for example as the distance in the $z$ axis between the two peaks in the energy density. For large $l$ the wall has a three layers structure shown in Fig. 2 in the first layer $\phi_{s}=\phi_{s 0}$ jumps to zero while $\phi_{\chi}=\phi_{\chi 0}$ is almost constant; in the second layer $\phi_{s}=0$ and $\phi_{\chi}$ goes to the value $\phi_{\chi}=-\phi_{\chi 0}$; in the third layer $\phi_{s}$ jumps to the value of $\phi_{s}=-\phi_{s 0}$. A more careful analysis of the energy density profile reveals always the presence of a third peak in the energy density just in between the other two symmetric walls in correspondence of the origin $\phi_{s}, \phi_{\chi}=0$ (see Fig. 3). The energy density evaluated at the origin diverges ${ }^{1}$. Note that the
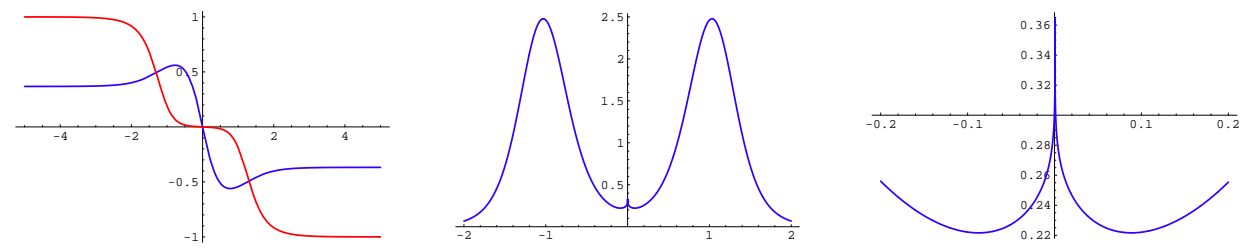

Figure 3: Wall for the Kähler potential $h_{2}$ and intermediate $l$ with respect to the previous figures. Left panel: Field profiles for $\phi_{s}(z)$ and $\phi_{\chi}(z)$. Central panel: Energy density. Right panel: Zoom of the central region which shows the presence of another energy peak at the center.

superpotential is regular near the origin.

The distance $l$ is a good parameter for the moduli space only in the limit in which the relative displacement of the two symmetric peaks of the energy density with respect to the origin is large. As can be seen in Fig 4 the two symmetric peaks in the energy density merge with the one at the origin in the limit of a small relative distance. A more precise way to parameterize

${ }^{1}$ The energy density of the BPS wall is given by the following expression:

$$
\rho=2\left|\frac{d}{d z} \operatorname{Re}(W)\right|=2\left|\frac{d \phi_{s}}{d z} \frac{\partial W}{\partial \phi_{s}}\right|+2\left|\frac{d \phi_{\chi}}{d z} \frac{\partial W}{\partial \phi_{\chi}}\right| .
$$

We also have near the origin at $\phi_{s}, \phi_{\chi}=0$ :

$$
\frac{\partial W}{\partial \phi_{\chi}} \approx \frac{\phi_{s}}{\phi_{\chi} \log \phi_{\chi}^{2}}, \frac{\partial W}{\partial \phi_{s}} \approx \log \left(\frac{\phi_{s}}{\phi_{\chi} \log \phi_{\chi}^{2}}\right) .
$$

The only way one can avoid a divergence is by requiring that $\frac{\phi_{s}}{\phi_{\chi} \log \phi_{\chi}^{2}}$ approaches a nonzero constant. This does not happen here since $\phi_{s} \approx C \phi_{\chi}$. 

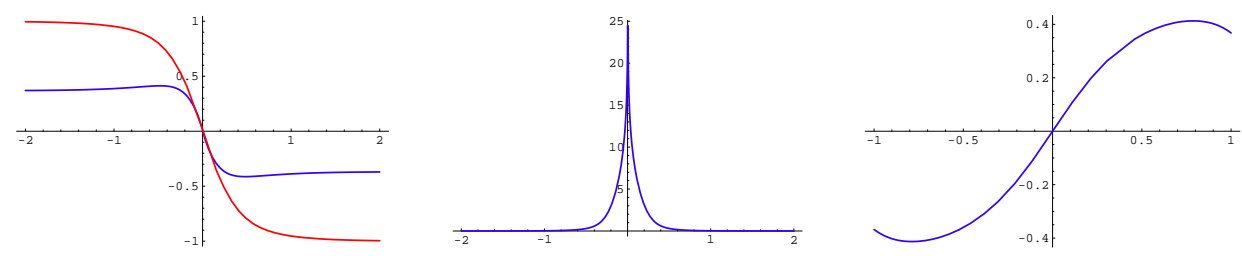

Figure 4: Wall for the Kähler potential $h_{2}$ and with $l$ small enough that the domain walls fuse in a single one. Left: Field profiles for $\phi_{s}(z)$ and $\phi_{\chi}(z)$. Center: Energy density. Right: Field profiles in the plane $\left(\phi_{s}, \phi_{\chi}\right)$.

the solutions is given by the real and positive constant $C$ introduced in (16):

$$
C=\lim _{\phi_{s}, \phi_{\chi} \rightarrow 0} \frac{\phi_{s}}{\phi_{\chi}} .
$$

In the limit $C \rightarrow 0$ the distance $l$ goes to infinity. For $C=\mathcal{O}(1)$ the two peaks in the energy density join with the third peak at the origin.

In order to have a better understanding of our results and provide a more precise relation between the hidden modulus $l$ and $C$ we have performed a

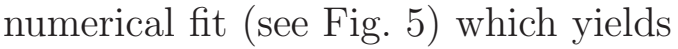

$$
\frac{l(C)}{2}=(0.14 \pm 0.03)+\frac{0.298 \pm 0.002}{C^{0.25}},
$$

where the errors are statistical in nature.



Figure 5: Fit providing the behavior of $l / 2$ as function of $C$. The solid curve corresponds to the fitted curve 20 reported in the main text. The dots are some representative points.

The situation looks similar to another model discussed in Ref. [15, 16]: 
this is a two superfield model with superpotential:

$$
W\left(\Phi_{1}, \Phi_{2}\right)=\frac{m^{2}}{\lambda} \Phi_{1}-\frac{1}{3} \lambda \Phi_{1}^{3}-\alpha \Phi_{1} \Phi_{2}^{2}
$$

and a standard Kähler potential:

$$
K=\Phi_{1} \bar{\Phi}_{1}+\Phi_{2} \bar{\Phi}_{2}
$$

There are four degenerate vacua in this model:

$$
\phi_{1}= \pm \frac{m}{\lambda}, \phi_{2}=0 \quad \text { and } \quad \phi_{1}=0, \phi_{2}= \pm \frac{m}{\sqrt{\lambda \alpha}}
$$

A family of degenerate BPS domain walls exists interpolating the two vacua with $\phi_{2}=0$ : this is due to the fact that this wall can be thought as composite of two walls interpolating between the $\phi_{2}=0$ vacua and the $\phi_{1}=0$ one. The relative distance of the two elementary and symmetric walls with respect to the origin is a modulus of this family of degenerate walls. There are similar composite walls also in $\mathcal{N}=2 U(N)$ SQCD in the Higgs phase with a FayetIliopolous term (see for example [17]).

The walls in the extended VY are somehow different from the ones just mentioned above. In this case the solution passes through an entire valley of vacua at $\phi_{s}=0$ and not through just another vacuum state.

\section{Issue of complex walls}

Let us take $N=2$; if the field $S$ is integrated out, the following result for $W_{\text {eff }}(\chi)$ is found:

$$
W_{e f f}(\chi)=\frac{4}{3} e \chi \log \chi^{2}
$$

It is easy to see that it is impossible to avoid the log-cuts in the superpotential: there are cuts on the imaginary positive and negative axis, and the only way to avoid them is to pass through the origin. This problem is still present in the complete superpotential:

$$
W(S, \chi)=\frac{8}{3} S\left[\log _{(0)}\left(\frac{S}{-e \frac{\chi}{2} \log _{(0)} \chi^{2}}\right)-1\right] .
$$


Here too, the only way to avoid the cuts on the positive and negative imaginary axis is to pass through $\phi_{\chi}=0$; but if we do this keeping $\phi_{s} \neq 0$ the superpotential diverges. This argument is not a rigorous non-existence proof, but shows that there is no obvious way to find such solutions.

In the framework of the Taylor, Veneziano and Yankielowicz theory ([18]), which is supposed to be the effective theory for $\mathcal{N}=1 U(N)$ SQCD, this problem [11, 14, 19] does not appear. For example, in the two color and two flavor case (i.e. $N=2, N_{f}=1$ ) we have:

$$
W(S, M)=\frac{2}{3} S \log \frac{S M}{e \Lambda^{5}}+\frac{m}{2} M .
$$

Here $M$ is the mesonic degree of freedom. Note also that here the normalization of the superpotential is slightly different than the one used earlier. With such a superpotential it is possible to find solutions in which the phase of $S$ and the one of $M$ cancel each other in such a way that the branch cuts of the logarithm are avoided. This mechanism does not work in the extended VY theory, because of the presence of another logarithm inside the argument of the first logarithm.

\section{Conclusions}

In this work we have investigated the domain wall problem related to the extended VY theory proposed in Ref. [7]. No BPS domain wall solution has been found for the general case of complex walls. However real solutions exist [9] for an even number of colors connecting two vacua aligned with the origin. Surprisingly we have discovered the presence of a new hidden noncompact modulus for the real walls. Although the presence of an hidden modulus is interesting per se, unfortunately it does not match the expected spectrum of domain walls for $\mathcal{N}=1$ super Yang-Mills. As discussed in Ref. [3, 4, 15], a discrete spectrum of wall with multiplicity

$$
n_{k}=\frac{N !}{k !(N-k) !}
$$

is expected. No theorem forbids a description of the SYM walls via an effective theory of its composite states. An argument against this possibility was suggested in Ref. [23] in the large $N$ limit; however, as pointed out in Ref. [22], this argument can be avoided if the wall thickness scales as 
$1 / N$ at large $N$. Various models, more or less linked to SYM were also considered in the past to mimic such a behavior [22, 20]. A satisfactory effective description of these objects should explain the discrete spectrum in Eq. (27) while using fields directly linked to SYM ${ }^{2}$. We have shown that although the extended VY theory displays some domain wall solutions these are not the ones expected on theoretical grounds.

At this point one may argue that the physics of the SYM walls should be described not via confined objects such as gluino-balls or glue-balls but via a more subtle substructure.

\section{Acknowledgments}

We are grateful to P. Merlatti, M. Shifman, A. Vainshtein and F. Vian for discussions and comments.

The work of R.A. is supported in part by DOE grant DE-FG02-94ER408. The work of F.S. is supported by the Marie Curie Excellence Grant as team leader under contract MEXT-CT-2004-013510 and by the Danish Research Agency. F.S. thanks the William I. Fine Theoretical Physics Institute for the kind hospitality during the initial stages of this work.

\section{References}

[1] G. R. Dvali and M. A. Shifman, "Dynamical compactification as a mechanism of spontaneous supersymmetry breaking," Nucl. Phys. B 504 (1997) 127 arXiv:hep-th/9611213.

[2] G. R. Dvali and M. A. Shifman, "Domain walls in strongly coupled theories," Phys. Lett. B 396, 64 (1997) [Erratum-ibid. B 407, 452 (1997)] arXiv:hep-th/9612128.

[3] C. Vafa, "Superstrings and topological strings at large N," J. Math. Phys. 42, 2798 (2001) arXiv:hep-th/0008142; B. S. Acharya and

\footnotetext{
${ }^{2}$ In Ref. 24 domain walls were studied in the softly broken $\mathcal{N}=2$ theory. The breaking to $\mathcal{N}=1$ was achieved by adding a mass term for the multiplet in the adjoint representation. In this model the picture proposed to understand the domain wall structure is indeed a complex one; the domain wall connects a vacuum with a monopole condensate to one with a dyonic condensate. Only one wall is found and not two as expected; a conjecture in order to solve this problem is presented in Ref. [25].
} 
C. Vafa, "On domain walls of $\mathrm{N}=1$ supersymmetric Yang-Mills in four dimensions," arXiv:hep-th/0103011.

[4] A. Ritz, M. Shifman and A. Vainshtein, "Counting domain walls in N = 1 super Yang-Mills," Phys. Rev. D 66, 065015 (2002) arXiv:hep-th/0205083.

[5] A. Ritz, "A note on domain walls and the parameter space of $\mathrm{N}=1$ gauge theories," JHEP 0310, 021 (2003) arXiv:hep-th/0308144.

[6] G. Veneziano and S. Yankielowicz, "An Effective Lagrangian For The Pure N=1 Supersymmetric Yang-Mills Theory," Phys. Lett. B 113, 231 (1982).

[7] P. Merlatti and F. Sannino, "Extending the VenezianoYankielowicz effective theory," Phys. Rev. D 70, 065022 (2004) arXiv:hep-th/0404251.

[8] A. Feo, P. Merlatti and F. Sannino, "Information on the super Yang-Mills spectrum," Phys. Rev. D 70, 096004 (2004) arXiv:hep-th/0408214.

[9] P. Merlatti, F. Sannino, G. Vallone and F. Vian, "N=1 super YangMills domain walls via the extended Veneziano Yankielowicz theory," Phys. Rev. D 71, 125014 (2005) arXiv:hep-th/0502196.

[10] A. Kovner and M. A. Shifman, "Chirally symmetric phase of supersymmetric gluodynamics," Phys. Rev. D 56, 2396 (1997) arXiv:hep-th/9702174.

[11] A. Kovner, M. A. Shifman and A. Smilga, "Domain walls in supersymmetric Yang-Mills theories," Phys. Rev. D 56, 7978 (1997) arXiv:hep-th/9706089.

[12] B. Chibisov and M. A. Shifman, "BPS-saturated walls in supersymmetric theories," Phys. Rev. D 56 (1997) 7990 [Erratum-ibid. D 58 (1998) 109901] arXiv:hep-th/9706141.

[13] I. I. Kogan, A. Kovner and M. A. Shifman, "More on supersymmetric domain walls, N counting and glued potentials," Phys. Rev. D 57, 5195 (1998) arXiv:hep-th/9712046. 
[14] A. Smilga and A. Veselov, "Complex Bogomolnyi-Prasad-Sommerfield domain walls and phase transition in mass in supersymmetric QCD," Phys. Rev. Lett. 79, 4529 (1997) arXiv:hep-th/9706217; A. V. Smilga and A. I. Veselov, "Domain walls zoo in supersymmetric QCD," Nucl. Phys. B 515, 163 (1998) arXiv:hep-th/9710123; A. V. Smilga, "BPS domain walls in supersymmetric QCD: Higher unitary groups," Phys. Rev. D 58, 065005 (1998) arXiv:hep-th/9711032; A. V. Smilga and A. I. Veselov, "BPS and non-BPS domain walls in supersymmetric QCD with SU(3) gauge group," Phys. Lett. B 428, 303 (1998) arXiv:hep-th/9801142.

[15] M. A. Shifman, "Degeneracy and continuous deformations of supersymmetric domain walls," Phys. Rev. D 57, 1258 (1998) arXiv:hep-th/9708060; M. A. Shifman and M. B. Voloshin, "Degenerate domain wall solutions in supersymmetric theories," Phys. Rev. D 57, 2590 (1998) arXiv:hep-th/9709137.

[16] A. A. Izquierdo, M. A. G. Leon, W. G. Fuertes, M. de la Torre Mayado and J. M. Guilarte, "Degenerate BPS domain walls: Classical and quantum dynamics," arXiv:hep-th/0311057.

[17] D. Tong, "The moduli space of BPS domain walls," Phys. Rev. D 66, 025013 (2002) arXiv:hep-th/0202012; A. Hanany and D. Tong, "On monopoles and domain walls," arXiv:hep-th/0507140.

[18] T. R. Taylor, G. Veneziano and S. Yankielowicz, "Supersymmetric QCD And Its Massless Limit: An Effective Lagrangian Analysis," Nucl. Phys. B 218 (1983) 493.

[19] B. de Carlos and J. M. Moreno, "Domain walls in supersymmetric QCD: From weak to strong coupling," Phys. Rev. Lett. 83, 2120 (1999) arXiv:hep-th/9905165; B. de Carlos, M. B. Hindmarsh, N. McNair and J. M. Moreno, "Domain walls in supersymmetric QCD," Nucl. Phys. Proc. Suppl. 101, 330 (2001) arXiv:hep-th/0102033.

[20] B. de Carlos, M. B. Hindmarsh, N. McNair and J. M. Moreno, "BPS domain walls in super Yang-Mills and Landau-Ginzburg models," JHEP 0108, 056 (2001) arXiv:hep-th/0106243. 
[21] G. Gabadadze, "The discrete $\mathrm{Z}(2 \mathrm{~N}(\mathrm{c}))$ symmetry and effective superpotential in SUSY gluodynamics," Nucl. Phys. B 544, 650 (1999) arXiv:hep-th/9808005.

[22] G. R. Dvali, G. Gabadadze and Z. Kakushadze, "BPS domain walls in large N supersymmetric QCD," Nucl. Phys. B 562, 158 (1999) arXiv:hep-th/9901032.

[23] E. Witten, "Branes and the dynamics of QCD," Nucl. Phys. B 507, 658 (1997) arXiv:hep-th/9706109.

[24] V. S. Kaplunovsky, J. Sonnenschein and S. Yankielowicz, "Domain walls in supersymmetric Yang-Mills theories," Nucl. Phys. B 552, 209 (1999) arXiv:hep-th/9811195.

[25] M. Shifman and A. Yung, "Domain walls and flux tubes in $\mathrm{N}=$ 2 SQCD: D-brane prototypes," Phys. Rev. D 67, 125007 (2003) hep-th/0212293. 